\title{
Unusual Presentation of Cystic Liver Metastases from Nasopharyngeal Carcinoma
}

\author{
Chien-Chang Kao ${ }^{a} \quad$ Cheng-Ping Yu ${ }^{b} \quad$ Yao-Chi Liu ${ }^{a} \quad J y h-C h e r n g ~ u^{a} \quad$ Chung-Bao Hsieh ${ }^{a}$ \\ a Department of Surgery,

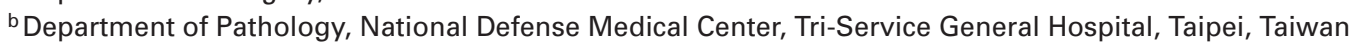

Dear editors,

In January 2005, a 35-year-old woman presented with a 2month history of enlarged nodules located on the right side of the neck and numbness of the right face. Nasopharyngeal endoscopy showed a bulging mass over the right Rosenmüller's fossa, and a biopsy was performed. The histological diagnosis revealed nasopharyngeal carcinoma (NPC) of an undifferentiated type. Magnetic resonance imaging of the nasopharynx and neck showed an infiltrating tumor invading into the bilateral locoregional lymph nodes, skull base, and C-spines. A whole-body bone scan confirmed the NPC with bone metastases. There were no pathological findings on abdominal ultrasound. The stage of NPC was determined to be pT4N2M1, stage IVc, Eastern Cooperative Oncology Group (ECOG): 0, VAS: 1 . The patient was treated by local radiation therapy combined with chemotherapy with cisplatin, 5-fluorouracil. 18 courses of these chemotherapeutic agents were administered. 1 year later, an abdominal sonogram showed multiple cystic lesions on the right liver lobe, and liver metastases were suspected. The chemotherapeutic agents were changed to cisplatin and docetaxel due to prior poor response. Over the next 6 months, the patient complained of abdominal fullness, poor appetite, and general weakness. She was then admitted to the hospital for further treatment. During hospitalization, physical examinations showed an ovoid shape of the abdomen with tenderness. Laboratory data showed: hemoglobin, $9.2 \mathrm{~g} / \mathrm{dl}$;

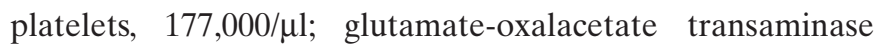
(GOT), $128 \mathrm{U} / \mathrm{l}$; glutamate-pyruvate transaminase (GPT), 63 U/l; total bilirubin, $1.9 \mathrm{mg} / \mathrm{dl}$; alkaline phosphatase, $442 \mathrm{U} / \mathrm{l}$; albumin, $2.8 \mathrm{~g} / \mathrm{dl}$. A computed tomography scan of the abdomen revealed hepatomegaly with multiple huge cystic lesions over both lobes of the liver (maximal one: $16 \times 17 \times 19 \mathrm{~cm}$ ) and one cystic lesion on the spleen (fig. 1). Liver aspiration with fluid cytology was performed. Histological examination showed hyperchromatic cells with oval to spindle shapes arranged in a sheet-like pattern with pleomorphism, a high nucleus/ cytoplasm (N/C) ratio, and a single distinct nucleolus. Associated infiltration of lymphoplasma cells was also seen. The evidence of undifferentiated NPC was supported by positive cytokeratin-18 and latent membrane protein-1 (LMP1) histochemical stains (fig. 2). Intermittent drainage of the hepatic cystic lesions for abdominal decompression was done, and adequate analgesia with psychological treatment was performed continuously. The patient eventually died from hepatic failure (total bilirubin, 24.7 mg/dl; GOT, 295 U/l; GPT, 92 U/l; alkaline phosphatase, $612 \mathrm{U} / 1)$.

In various reports, the incidence of distant metastases from NPC ranges from 17 to 54\% [1]. Bone, lung, liver, and distant lymph nodes are the most common sites of distant metastases [2]. Liver is the third most frequent site of metastases with an incidence of $29.3-38 \%$ [1,2]. Cystic liver metastases are rare; they usually derive from colorectal cancer [3] and rarely from pancreatic, gastric, or lung cancer [4]. The cause of the cystic change in these secondary liver tumors is not well understood. It is believed that the hemorrhage and necrosis usually present in these lesions indicate that the tumor has grown rapidly, outstripping/depleting its blood supply [5]. NPC with liver invasion usually presents solitary or multiple solid tumor metastases. Metastatic cystic hepatic lesions deriving from NPC have not been reported before, as it is difficult to distinguish these from other cystic hepatic lesions. An accurately defined diagnosis by a computed tomogram or ultrasound is not easy, and histological confirmation is necessary. Therefore, fluid cytology plus cell block by liver cyst aspiration is recommended to be performed obligatorily. Concurrent chemoradiotherapy is the standard treatment for the primary tumor site and the metastatic NPC [6]. The distant metastasis in NPC has remained the major cause of mortality and treatment failure despite better locoregional control of the disease [2]. In the case reported here, chemotherapy had a poor response in the pa-

\section{KARGER}

Fax +497614520714

Information@Karger.de

www.karger.com
(C) 2008 S. Karger GmbH, Freiburg

Accessible online at:

www.karger.com/onk
Chung-Bao Hsieh, MD

Division of General Surgery, Tri-Service General Hospital

No. 325, Cheng-Kung Road, Sec. 2

Neihu 114, Taipei, Taiwan, R.O.C

Tel. +886 2 87927191, Fax -273

jogger0836@pchome.com.tw 


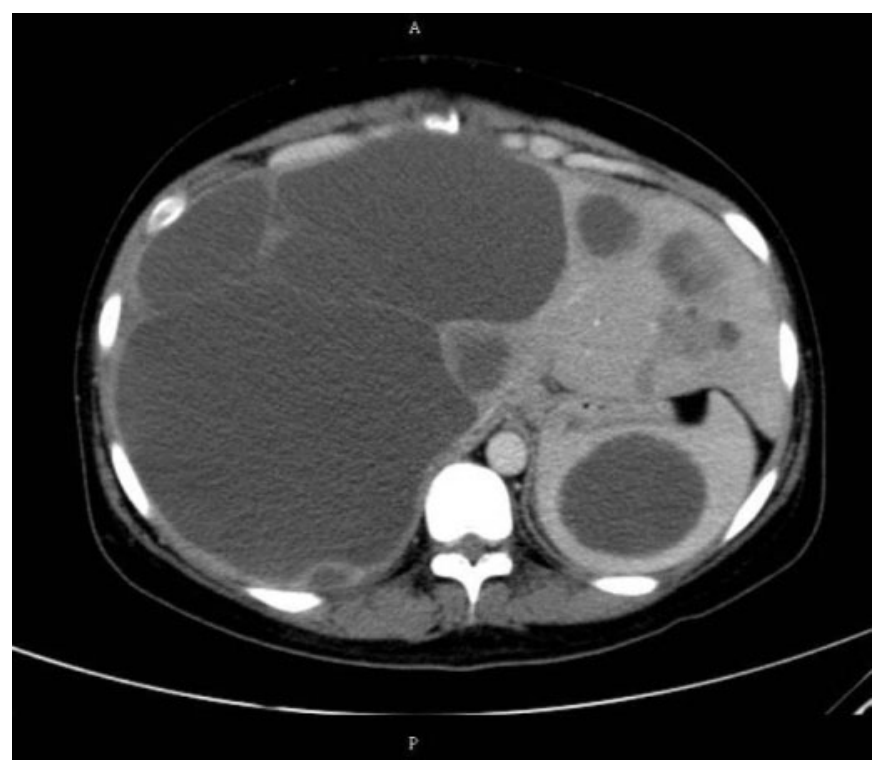

Fig. 1. Multiple huge cystic lesions occupied both lobes of the liver and one cystic lesion was located in the spleen.

tient, with even distant metastases spreading rapidly. The liver parenchyma was almost consumed by metastatic NPC. Impaired liver function with jaundice followed. Finally, the patient died of hepatic coma with multiple organ failure. In conclusion, the prognosis of NPC with development of distant

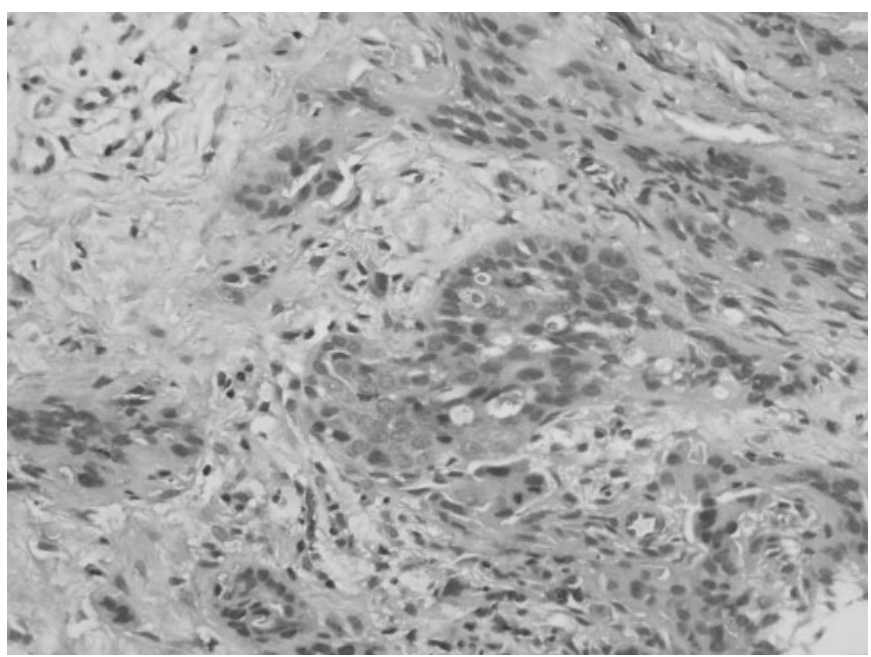

Fig. 2. Many hyperchromatic cells with oval to spindle shapes were arranged in a sheet-like pattern with pleomorphism, a high N/C ratio, and a single distinct nucleolus (hematoxylin \& eosin stain, $\times 100$ ).

metastases is poor. Once adjuvant chemotherapy has failed to control tumor metastases, the patient's life expectancy is short. Therefore, palliative treatment is important in reducing the patient's discomfort and gaining quality of life.

\section{References}

1 Ahmad A, Stefani S: Distant metastases of nasopharyngeal carcinoma: A study of 256 male patients. J Surg Oncol 1986;33:194-197.

$\checkmark 2$ Chih-Jen H, Stephen WL, Shi-Long L, Chong-Jong W, Fu-Min F, Yau-Hui H: Patterns of distant metas tases in nasopharyngeal carcinoma. Kaohsiung $\mathrm{J}$ Med Sci 1996;12:229-234.
Suawara Y, Yamamoto J, Yamasaki S, Shimada K, Kosuge T, Sakamoto M: Cystic liver metastases from colorectal cancer. J Surg Oncol 2000;74:148152.

4 Fabrizio R, Andrea P, Roberto C, Fabio U, Matteo $\mathrm{C}$, Franco U: Cystic liver metastases from lung adenocarcinoma: a case report. Tumori 2004;90:525527.
5 Aoki K, Takayasu K, Muramatsu Y: Spontaneous massive hemorrhage within a malignant tumor of the liver: diagnostic features in sonography and CT. Jpn J Clin Oncol 1991;21:366-371.

6 Fuchs S, Rödel C, Brunner T, Iro H, Niedobitek G, Sauer R, Grabenbauer RR: Patterns of failure following radiation with and without chemotherapy in patients with nasopharyngeal carcinoma. Onkologie 2003;26;12-18. 